\title{
Urea Permeability of Mammalian Inner Medullary Collecting Duct System and Papillary Surface Epithelium
}

Jeff M. Sands and Mark A. Knepper

Laboratory of Kidney and Electrolyte Metabolism, National Heart, Lung and Blood Institute, National Institutes of Health, Bethesda, Maryland 20892

\begin{abstract}
To compare passive urea transport across the inner medullary collecting ducts (IMCDs) and the papillary surface epithelium (PSE) of the kidney, two determinants of passive transport were measured, namely permeability coefficient and surface area. Urea permeability was measured in isolated perfused IMCDs dissected from carefully localized sites along the inner medullas of rats and rabbits. Mean permeability coefficients $\left(\times 10^{-5} \mathrm{~cm} / \mathrm{s}\right)$ in rat IMCDs were: outer third of inner medulla $\left(I M C D_{1}\right), 1.6 \pm 0.5$; middle third $\left(\mathrm{IMCD}_{2}\right), 46.6 \pm 10.5$; and inner third $\left(\mathrm{IMCD}_{3}\right)$, 39.1 \pm 3.6. Mean permeability coefficients in rabbit IMCDs were: IMCD, $1.2 \pm 0.1 ; \mathrm{IMCD}_{2}, 11.6 \pm 2.8$; and $\mathrm{IMCD}_{3}, 13.1 \pm 1.8$. The rabbit PSE was dissected free from the underlying renal inner medulla and was mounted in a specially designed chamber to measure its permeability to urea. The mean value was $1 \times 10^{-5}$ $\mathrm{cm} / \mathrm{s}$ both in the absence and presence of vasopressin (10 $\mathrm{nM}$ ). Morphometry of renal papillary cross sections revealed that the total surface area of IMCDs exceeds the total area of the PSE by 10-fold in the rat and threefold in the rabbit. We conclude: (a) the IMCD displays axial heterogeneity with respect to urea permeability, with a high permeability only in its distal twothirds; and (b) because the urea permeability and surface area of the PSE are relatively small, passive transport across it is unlikely to be a major source of urea to the inner medullary interstitium.
\end{abstract}

\section{Introduction}

Urea is the chief end product of nitrogen metabolism in mammals. It is a major osmotic component of the urine, typically accounting for $20-60 \%$ of the total urinary osmolality in humans, depending on the protein content of the diet (1). Its concentration in the urine typically exceeds $1 \mathrm{M}$ during antidiuresis, i.e., when water excretion is low in response to high circulating levels of vasopressin. Chemical analysis of renal tissue from dogs (2), rats (3), and rabbits (4), and micropuncture measurement of urea concentrations in vasa recta plasma of rodent inner medulla (5-8) have demonstrated that urea accumulates in the inner medullary tissue to concentrations almost as high as in the final urine.

Urea accumulation in the inner medullary interstitium is critical to renal water conservation. The large amount of urea excreted in the urine would be expected to retard water absorption from the collecting ducts by its osmotic effect. However, as

Address correspondence to Dr. Knepper, Building 10, Room 6N307, National Institutes of Health, Bethesda, MD 20892.

Received for publication 4 June 1986.

The Journal of Clinical Investigation, Inc.

Volume 79, January 1987, 138-147 originally pointed out by Berliner and his colleagues (9), the high urea concentration in the inner medullary interstitium balances the osmotic effect of urea in the lumens of the inner medullary collecting ducts. This allows continued water absorption from the collecting ducts driven by the medullary $\mathrm{NaCl}$ gradient. Furthermore, it has been proposed that urea accumulation in the inner medullary interstitium may play a role in the passive generation of the inner medullary $\mathrm{NaCl}$ concentration gradient $(10,11)$.

Accumulation of urea in the inner medullary interstitium requires a continuous source of urea from one or more renal tubular or vascular structures. A major source is the inner medullary collecting ducts. Microcatheterization studies in hamsters (12) and micropuncture studies in rats (13) have demonstrated high rates of urea absorption from inner medullary collecting ducts, amounting to $15-30 \%$ of the filtered load. Two observations support the hypothesis that urea absorption from the inner medullary collecting duct is largely passive. First, under normal nondiuretic conditions in rats, the luminal urea concentration in the inner medullary collecting ducts exceeds that in neighboring vasa recta, providing a concentration gradient favorable for passive absorption $(5,7)$. Second, permeability measurements in rat inner medullary collecting ducts have demonstrated a very high passive urea permeability, which is increased further by vasopressin $(14,15)$.

Controversy has arisen, however, from permeability measurements in rabbit collecting ducts. The urea permeability of isolated inner medullary collecting ducts from rabbits (16) was found to be an order of magnitude lower than values measured in inner medullary collecting ducts from rats $(14,15)$. Rector (17) has questioned whether this permeability is sufficient to allow adequate rates of urea absorption from rabbit inner medullary collecting ducts to replace urea lost via the vasa recta and loop of Henle. He raised the possibility that another source, such as the renal pelvic urine, supplies the urea that accumulates in the inner medullary interstitium of rabbits. However, anatomical studies of the inner medullary collecting ducts in rats and rabbits offer a different explanation for the difference in measured urea permeability in rat and rabbit inner medullary collecting ducts. These studies have demonstrated major changes in the morphological appearance of the inner medullary collecting duct along its length (18-20). The demonstrated morphological heterogeneity along the inner medullary collecting duct raises the possibility that there may be functional heterogeneity as well. These considerations led us to the hypothesis that the difference in the measured urea permeability in rabbit and rat inner medullary collecting ducts was due to a difference in the origin of the tubules along the inner medullary axis rather than to a basic difference between rat and rabbit kidneys. The first objective of the present study was to test this hypothesis by measuring the urea permeability of isolated inner medullary 
collecting ducts dissected from carefully localized sites along the entire inner medullary axis of both rats and rabbits.

In addition to the inner medullary collecting ducts, a second source of urea transfer to the inner medullary interstitium has been proposed, namely the renal pelvis (21-25). Urine exiting the inner medullary collecting ducts at the tip of the renal papilla enters the pelvic space where it bathes the outer surface of the papilla. It has been proposed that urea in the renal pelvic urine can passively penetrate the epithelium that covers the papilla (the papillary surface epithelium) and enter the inner medullary interstitium, thus contributing to inner medullary urea accumulation. Assessment of the quantitative importance of urea transport from the pelvic urine to the inner medullary interstitium requires knowledge of the permeability of the epithelium to urea. Therefore, the second objective of the present study was to measure the permeability of the rabbit papillary surface epithelium to urea. To do this, we have developed a method for isolating the papillary surface epithelium from the underlying inner medulla of the rabbit and studying it in vitro using a specially designed chamber.

In addition to permeability, another important determinant of passive urea transport is epithelial surface area. The rate of urea entry into the inner medullary interstitium from the inner medullary collecting ducts and from the renal pelvic space depends in part on the surface areas of the two epithelial barriers. To allow a quantitative comparison of urea transport across inner medullary collecting ducts and papillary surface epithelium, the third objective of the present study was to measure the total epithelial surface area of these two epithelia in the renal papilla. To do this, we have used standard morphometric techniques to analyze histologic cross sections from rat and rabbit renal papillas.

\section{Methods}

Isolated perfused tubule experiments. Pathogen-free male Sprague-Dawley rats weighing 65-140 g (Small Animal Breeding Facility, National Institutes of Health, Bethesda, MD) and pathogen-free female rabbits weighing 1,200-2,000 g (Small Animal Breeding Facility) were used for the studies. The animals were injected with furosemide $(1 \mathrm{mg} / 100 \mathrm{~g}$ body wt i.p. in rats; $1 \mathrm{mg} / 200 \mathrm{~g}$ body wt i.p. in rabbits) $20 \mathrm{~min}$ before decapitation. This resulted in a rapid diuresis and presumably washed out most of the inner medullary axial solute concentration gradient present in untreated animals. This allowed the kidneys to be removed from the animals and to be placed in chilled isotonic dissection medium (described below) without danger of osmotic shock to the cells. Preliminary observations in rats demonstrated that this procedure greatly facilitates the dissection allowing undamaged tubules to be obtained from virtually every animal killed.

Coronal slices were cut from the kidneys and were transferred to a dissection dish containing chilled dissection solution for isolation of inner medullary collecting ducts. The dissection solution contained (in millimoles): $\mathrm{NaCl}, 124 ; \mathrm{NaHCO}_{3}, 25 ; \mathrm{K}_{2} \mathrm{HPO}_{4}, 2 ; \mathrm{CaCl}, 2 ; \mathrm{MgSO}_{4}, 1.2 ;$ glucose, 5.5; and urea, 5 . It was gassed continuously with $95 \%$ air and $5 \% \mathrm{CO}_{2}$ before and during the dissection. The dissection medium was maintained at $17^{\circ} \mathrm{C}$ by a transparent polymethylmethacrylate heat exchanger inserted between the dissection dish and the stage of the dissection microscope (model M-8; Wild Heerbrugg Instruments Inc., Farmingdale, NY). The heat exchanger was cooled by a refrigerated circulator. The dissection was done using diffuse lighting transmitted from the stage below the dish rather than with darkfield illumination, which is more commonly used in isolated perfused tubule studies. In preliminary studies, it was found that the inner medullary collecting ducts are only poorly visualized under darkfield illumination.
Inner medullary collecting ducts were dissected from carefully localized positions along the inner medullary axis. To do this, the total length of the inner medulla from the inner-outer medullary junction to the papillary tip was first measured using a calibrated eyepiece micrometer. Then the intended location of the proximal-most end of the perfused tubule was marked by crimping the tissue with the dissection forceps (Dumont No. 5). In this manner, the proximal end of the dissected tubule could be localized with an accuracy of $\sim \pm 5 \%$ of the total inner medullary length.

The tubules were mounted on concentric pipettes as described previously (26) and were studied at $37^{\circ} \mathrm{C}$. The perfusion and bath solutions were identical to the dissection medium (described above) except that raffinose $(5 \mathrm{mM})$ replaced urea in either the perfusate or bath. Thus a bath-to-lumen or lumen-to-bath urea concentration difference was present establishing a gradient driving the passive transport of urea. The urea transport rate $\left(J_{\mathrm{u}}\right)$ was calculated as: $J_{\mathrm{u}}=C_{0} \cdot V_{0}-C_{\mathrm{L}} \cdot V_{\mathrm{L}}$, where $C_{0}$ is the urea concentration in the perfusate, $C_{\mathrm{L}}$ is the urea concentration in the collected fluid, $V_{0}$ is the perfusion rate per unit length, and $V_{\mathrm{L}}$ is the rate of fluid flow exiting the tubule per unit length. The permeability $\left(P_{u}\right)$ was calculated from the urea transport rate $\left(J_{u}\right)$ using the equation: $P_{\mathrm{u}}=J_{\mathrm{u}} /(\pi \cdot \mathrm{D} \cdot \delta \mathrm{C})$, where $\delta \mathrm{C}$ is the mean urea concentration difference along the tubule and $\mathrm{D}$ is the tubule inner diameter measured from Polaroid photographs of the perfused tubule. In a few experiments, urea permeability was determined with a 100 -mM bath-to-lumen urea concentration gradient using $100 \mathrm{mM}$ urea in the bath and $100 \mathrm{mM}$ raffinose in the lumen for osmotic balance.

In additional experiments, the permeability of inner medullary collecting ducts to creatinine was determined by measuring the creatinine flux in the presence of a 5-mM bath-to-lumen creatinine concentration gradient. In these experiments, creatinine replaced urea in the bath solution, but the conditions of study were otherwise identical to those used for the urea permeability measurements.

The urea concentrations in perfusate, bath, and collected fluid were measured using a continuous-flow ultramicro-fluorometer similar to that previously described for measurement of total ammonia in nanoliter volume samples (27). The urea method uses two enzymatic reactions: (a) conversion of urea to ammonia catalyzed by urease, and $(b)$ reaction of the ammonia with $\alpha$-ketoglutarate and NADH to form NAD, catalyzed by glutamate dehydrogenase. The reagents were purchased as a kit (kit 65-A; Sigma Chemical Co., St. Louis, MO). The disappearance of NADH (measured as a reduction from the baseline fluorescent signal) is proportional to the urea present in the injected sample. Fig. 1 shows a stan-

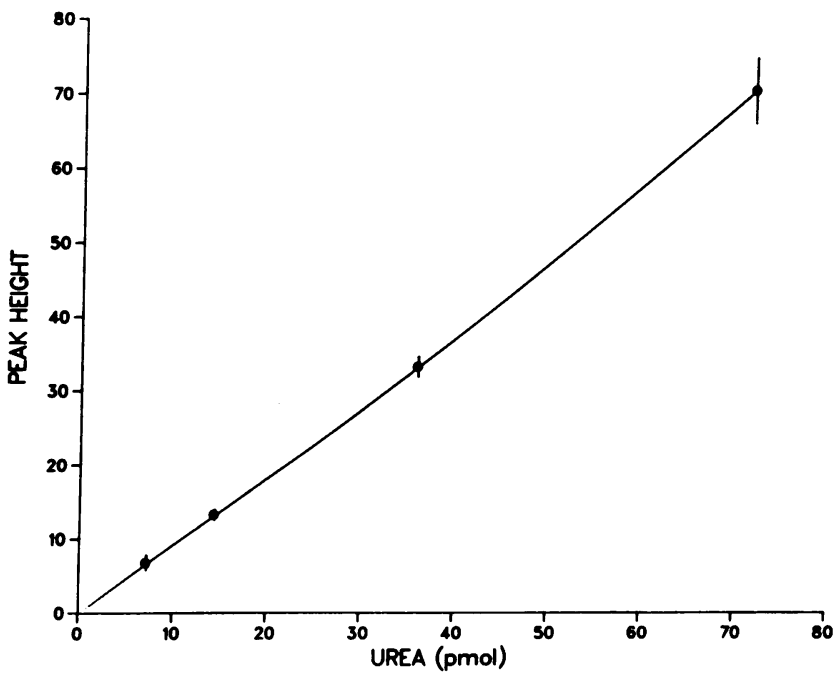

Figure 1. Standard curve for fluorometric urea method. Each point shows mean \pm SD for five determinations. A 36-nl constriction pipet was used to inject standards containing $0.2,0.4,1.0$, and $2.0 \mathrm{mM}$ urea. 
dard curve for the method. The method is linear from 0 to $60 \mathrm{pmol}$ urea and is capable of resolving differences of $4 \%$ or greater in urea content between samples in the size range used in experiments (12-36 pmol).

The creatinine concentrations in perfusate, bath, and collected fluid were measured using a continuous-flow colorimeter as previously described (28). The reagents for the determinations were purchased as a kit (kit 555-A; Sigma Chemical Co.). The method is linear up to 150 pmol creatinine and is capable of resolving differences of 3\% or more between samples in the size range 50-150 pmol. The transepithelial potential difference was measured as previously described (29).

Isolated papillary surface epithelium studies. Both kidneys were removed from pathogen-free rabbits treated with furosemide as described above. Each kidney was cut with a scalpel blade so as to yield an intact papilla with a rectangular piece of cortex and outer medulla attached. Each papilla was then bisected along its long axis to yield two hemipapillas. Using a dissecting microscope (model M8; Wild Heerbrugg Instruments Inc.) and dissecting forceps (Dumont No. 5), the collecting ducts and other structures were dissected from the interstitial side of a hemi-papilla, leaving the papillary surface epithelium isolated as a flat sheet with a $20-50 \mu \mathrm{m}$ underlying layer of inner medullary tissue consisting of interstitial matrix, nephrons, and blood vessels. The attached cortex and outer medulla were used to hold the tissue with the left-hand pair of forceps while the dissection was done with the right-hand pair of forceps. After completion of the papillary dissection, the attached cortex and outer medulla were cut free before placing the tissue in a specially designed chamber for transport studies (described below).

The basic dissection and perfusion solution contained (in millimoles): $\mathrm{NaCl}, 118 ; \mathrm{NaHCO}_{3}, 25 ; \mathrm{K}_{2} \mathrm{HPO}_{4}, 2.5 ; \mathrm{CaCl}_{2}, 2 ; \mathrm{MgSO}_{4}, 1.2$; glucose, 5; urea, 100; and creatinine, 5. This solution was used to bathe both the apical and basolateral surfaces of the epithelium during experiments. Either carboxy $\left[{ }^{14} \mathrm{C}\right]$ inulin or $\left[{ }^{14} \mathrm{C}\right]$ urea was added to the apical solution. In some experiments, arginine vasopressin $\left(10^{-8} \mathrm{M}\right.$; Sigma Chemical Co.) was added to the basolateral perfusion solution.

Fig. 2 illustrates the chamber design. The area of the aperture was 7 $\mathrm{mm}^{2}$. The tissue was mounted on eight steel pins (not shown in Fig. 2) extending upward from the bottom half of the chamber. The periphery of the tissue was supported on top and bottom by thin washers made of hardened Sylgard (silicone elastomer No. 184, Dow Corning Corp., Midland, MI). A water-tight seal between the Sylgard washers and the tissue, and between the Sylgard washers and the surfaces of the Lucite chamber was achieved by coating the Sylgard washers with a thin layer of liquid Sylgard before mounting the tissue. This method of sealing the tissue in the chamber was modelled after that described by Helman et al. (30). The two halves of the chamber were gently secured with three screws. Because the peripheral portions of the tissue were used for mounting the tissue, the only part of the papillary surface epithelium exposed in this preparation was that originally covering the middle third of the papilla.

The apical side of the tissue was covered by an aqueous droplet (100 $\mu \mathrm{l})$, which in turn was covered by water-equilibrated mineral oil. The entire chamber was submerged in water-equilibrated mineral oil which was maintained at $37^{\circ} \mathrm{C}$ in a temperature-controlled water bath. The basolateral half of the chamber was perfused continually at $0.8 \mathrm{ml} / \mathrm{min}$. The basolateral perfusate was heated to $37^{\circ} \mathrm{C}$ by passing the feed line through the heated mineral oil bath en route to its connection with the chamber input port. Both the mineral oil and the perfusion solutions were bubbled with $95 \%$ air and $5 \% \mathrm{CO}_{2}$ during the experiments. In technical control experiments using a glass $\mathrm{pH}$ microelectrode (model $\mathrm{M}$ 710 , Microelectrodes, Inc., Londonderry, $\mathrm{NH}$ ), the system was found to maintain the $\mathrm{pH}$ in the basolateral half of the chamber at 7.46-7.48. The temperature inside the chamber was found to be $37^{\circ} \mathrm{C}$ with a thermistor (400 series, Yellow Springs Instrument Co., Yellow Springs, $\mathrm{OH}$ ) in additional technical control experiments.

Transepithelial voltage and resistance were routinely measured in each experiment. Separate electrode pairs were used for voltage measurement and current injection. In the voltage circuit, calomel halfcells were connected to the apical and basolateral sides of the chamber by $160 \mathrm{mM} \mathrm{NaCl}$-agarose bridges. A high impedance electrometer (KS-

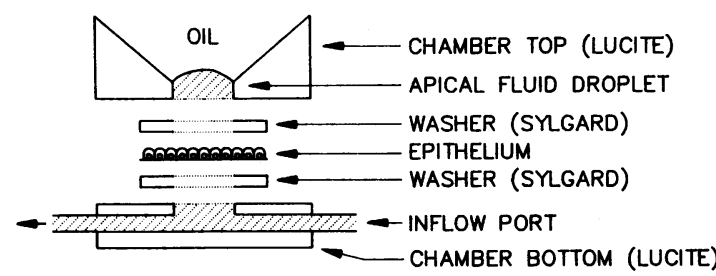

Figure 2. Chamber design. Chamber used for mounting isolated papillary surface epithelium. Tissue is mounted on eight pins (not shown) that extend from the Lucite chamber bottom. Aqueous solutions indicated by cross hatching. See Methods section for description of method used in mounting tissue and preventing leaks.

700; World Precision Instruments, Inc., New Haven, CT) was used and the output was continuously recorded using a standard chart recorder. In the current injection circuit, a $\mathrm{Ag}-\mathrm{AgCl}$ half cell (EH-1S; World Precision Instruments Inc.) was connected to the apical droplet via a 160 $\mathrm{mM} \mathrm{NaCl}$-agarose bridge and a $\mathrm{AgCl}$ wire was connected to the basolateral bath via the basolateral outflow port. A specially built constant current source (Dr. Roderic Steele, Laboratory of Technical Development, National Heart, Lung and Blood Institute) was used to inject current. The resistance of the epithelium was calculated using Ohm's law from the voltage deflection produced by injection of $\pm 1 \mu \mathrm{A}$ current. The output of the current source was measured using a digital multimeter (model 191; Keithley Instruments, Inc., Cleveland, OH).

The permeability $(P)$ of the epithelium to urea or inulin was calculated from the radioactivity in the basolateral output stream $\left(R_{0}\right)$ when radiolabeled urea or inulin was added to the apical droplet using the equation: $\mathrm{P}=R_{0} /\left(\mathrm{t} \cdot X_{\mathrm{a}} \cdot C_{\mathrm{a}} \cdot A_{\mathrm{s}}\right)$, where $\mathrm{t}$ is the collection time, $X_{\mathrm{a}}$ is the specific activity of the radionuclide in the apical droplet, and $C_{\mathrm{a}}$ is the chemical concentration of urea or inulin in the apical droplet, and $A_{s}$ is the surface area of the epithelium $\left(7 \mathrm{~mm}^{2}\right)$. The radioactivity of the basolateral output and apical droplet was measured using a liquid scintillation counter (LS350; Beckman Instruments, Inc., Fullerton, CA).

In preliminary experiments, the $\left[{ }^{14} \mathrm{C}\right]$ inulin permeability and electrical resistance were measured in several papillary epithelia to develop an electrical criterion for epithelial damage. The inulin permeability was low and independent of resistance above $63 \Omega \cdot \mathrm{cm}^{2}$. (mean permeability coefficient, $1.39 \pm 0.22 \times 10^{-6} \mathrm{~cm} / \mathrm{s}[n=9]$ ). At resistances below 60 $\Omega \cdot \mathrm{cm}^{2}$, the inulin permeability was at least an order of magnitude higher Based on these observations, a minimum resistance of $70 \Omega \cdot \mathrm{cm}^{2}$ was selected as the criterion for acceptance of an epithelium for further study. Throughout the study, $\sim 90 \%$ of epithelia placed in the chamber met this criterion. Previous studies of this epithelium using high resolution differential interference contrast microscopy of the living tissue demonstrated that an intact epithelium with undamaged cells can routinely be isolated (31).

Surface area measurements. To determine the relative epithelial surface areas of inner medullary collecting ducts and the papillary surface epithelium in the renal papilla, histological cross sections of rat and rabbit papillas were analyzed morphometrically. The tissue sections were prepared as part of a previous morphometric study of the renal medulla (18). In brief, the animals were anesthetized, a mannitol diuresis was established, the renal vein was clamped, the kidney was resected, and the entire kidney was fixed by immersion in chilled $10 \%$ formalin. $5-\mu \mathrm{m}$ sections were made perpendicular to the papillary axis and stained with hematoxylin and eosin.

The morphometric analysis was carried out only for the papillary portion of the inner medulla, i.e., the conelike projection of the inner medulla into the pelvic space. Operationally, this meant that the analysis was restricted to sections of inner medullary tissue that were completely surrounded by the pelvic space. To determine the perimeter and crosssectional area of each papillary section, the entire circumference of each section was traced from projections made with a trinocular microscope fitted with a projecting prism (18). The magnification of the projections was determined by use of a stage micrometer. 


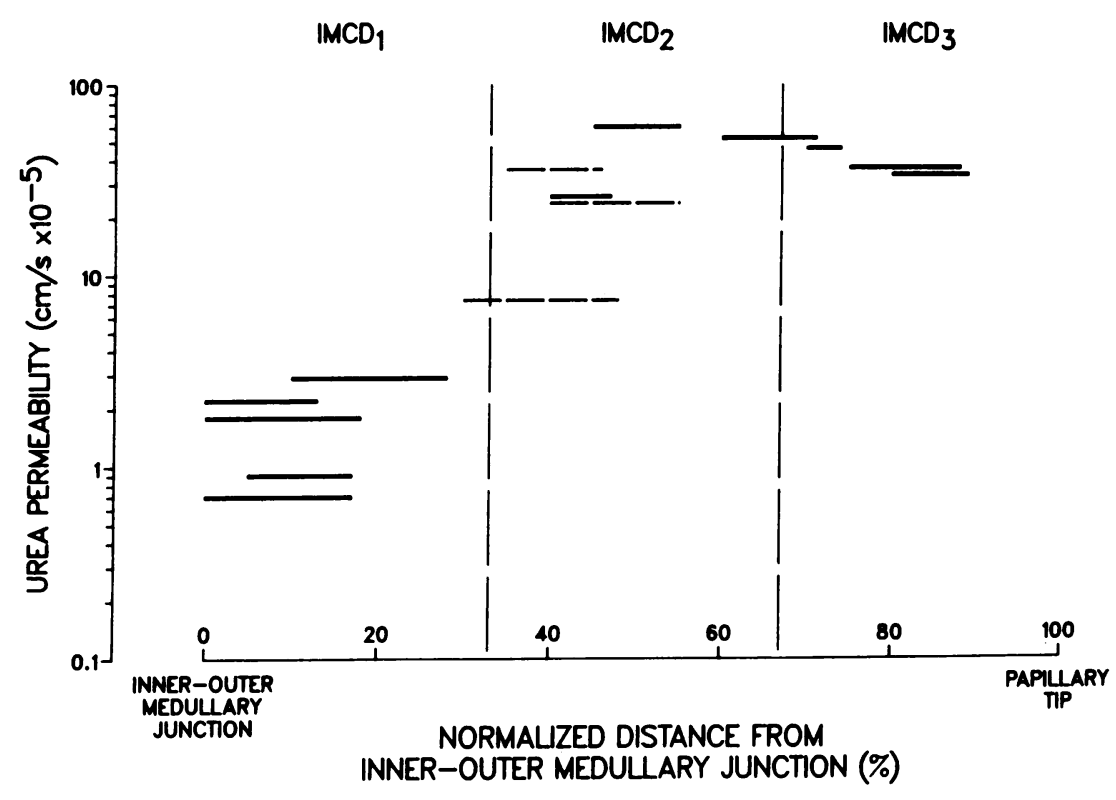

Figure 3. Rat inner medullary collecting ducts. Urea permeabilities in rat inner medullary collecting ducts as a function of distance along inner medullary axis. The mean permeability for each tubule is represented by a horizontal line that indicates the original position of the tubule in the inner medulla. Solid lines represent permeabilities measured with a 5-mM bath-to-lumen urea gradient. Dashed horizontal lines represent permeabilities measured with a 5-mM lumen-to-bath urea gradient. To allow statistical comparisons, tubules were arbitrarily divided into three groups based on the origin of the tubules in the medulla: $I M C D_{1}$, $\mathrm{IMCD}_{2}$, and $\mathrm{IMCD}_{3}$. Permeability in $\mathrm{IMCD}_{1}$ was significantly lower than in $\mathrm{IMCD}_{2}$ or $\mathrm{IMCD}_{3}$ by analysis of variance $(P<0.01$ using Bonferroni's method for contrasts). See Table I for full descriptive summary of experiments.
The surface area of the papillary surface epithelium per unit papillary length at each point along the papilla is equal to the perimeter of the papillary sections. The perimeter of each section was determined by measuring the length of the traced circumference of the projected image. The perimeter was plotted against distance of the section from the papillary tip. The area under this curve was estimated numerically using the trapezoidal rule (32) to obtain the total papillary surface area.

The total surface area of all inner medullary collecting ducts per unit papillary length is equal to the sum of the perimeters of all inner medullary collecting ducts at a given papillary level $\left(A_{\mathrm{i}}\right) . A_{\mathrm{i}}$ can be calculated from $A_{\mathrm{i}}=\mathrm{N} \cdot \pi \mathrm{D}$ where $\mathrm{N}$ is the total number of collecting ducts and $\mathrm{D}$ is the mean collecting duct diameter. We determined $\mathrm{N}$ by measuring the cross-sectional area of each papillary section and multiplying by the number of collecting ducts per unit cross-sectional area. The latter values were obtained from Knepper et al. (18). The cross-sectional area of each papillary cross section was measured by planimetry of the papillary projections. The total surface area of inner medullary collecting ducts in the papilla was then determined by integrating under the curve relating total perimeter $\left(A_{i}\right)$ to distance from the papillary tip using the numerical method described above.

\section{Results}

Urea permeability of rat inner medullary collecting ducts. The urea permeability coefficient was measured in 11 rat inner medullary collecting ducts dissected from defined points along the long axis of the inner medulla (Fig. 3). Table I gives a descriptive summary of these experiments and three additional experiments in cortical collecting ducts. To summarize the data and to carry out statistical comparisons, the inner medullary collecting duct was arbitrarily divided into thirds using the terminology proposed

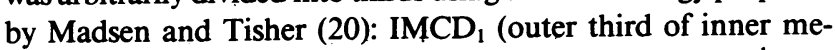
dulla), $\mathrm{IMCD}_{2}$ (middle third), and $\mathrm{IMCD}_{3}$ (inner third). ${ }^{1}$ The urea permeability was relatively low in $\mathrm{IMCD}_{1}$ and remarkably high in $\mathrm{IMCD}_{2}$ and $\mathrm{IMCD}_{3}$ (Fig. 3). It was necessary to perfuse IMCD 2 and $\mathrm{IMCD}_{3}$ segments at very rapid rates (Table I) to avoid complete equilibration of urea in the tubule fluid with the bath. As previously found (33), the urea permeability of rat cortical collecting ducts was very low (Table I).

1. Abbreviations used in this paper: $\mathrm{IMCD}_{1}, \mathrm{IMCD}_{2}$, and $\mathrm{IMCD}_{3}$, outer, middle, and inner third of inner medulla, respectively.
To determine whether the high urea permeability in tubules from the inner two-thirds of the inner medulla is associated with a generally high permeability to nonelectrolytes, the creatinine permeability of four inner medullary collecting ducts IMCD $_{2}$ and $\mathrm{IMCD}_{3}$ ) was determined. The creatinine permeability $\left(1.3 \pm 0.5 \times 10^{-5} \mathrm{~cm} / \mathrm{s}\right)$ was 33-fold lower than the average urea permeability for $\mathrm{IMCD}_{2}$ and $\mathrm{IMCD}_{3}\left(42.8 \times 10^{-5} \mathrm{~cm} / \mathrm{s}\right)$.

In all of the experiments described above, the urea permeability was determined by measuring the flux resulting from a bath-to-lumen concentration gradient. In vivo, the gradient is oriented in the opposite direction. Consequently, we carried out three additional experiments in $\mathrm{IMCD}_{2}$ using a lumen-to-bath urea concentration gradient to rule out any possible systematic error related to the direction of the gradient. The permeability values (shown as dashed horizontal lines in Fig. 3) are consistent with the values found with a bath-to-lumen gradient (solid horizontal lines).

Urea permeability was determined in three additional inner medullary collecting ducts ( $\mathrm{IMCD}_{2}$ segments) using a $100-\mathrm{mM}$ bath-to-lumen urea concentration gradient. The measured permeability $\left(27 \pm 4 \times 10^{-5} \mathrm{~cm} / \mathrm{s}\right)$ was not markedly different from the value determined with a $5 \mathrm{mM}$ concentration gradient in the same tubules $\left(38 \pm 9 \times 10^{-5} \mathrm{~cm} / \mathrm{s}\right)$.

Urea permeability of rabbit inner medullary collecting ducts. The urea permeability was measured in nine rabbit inner medullary collecting ducts dissected from defined points along the long axis of the inner medulla (Fig. 4). Table II gives a descriptive summary of these experiments. As Fig. 4 illustrates, the relationship between urea permeability and location along the inner medullary axis is qualitatively the same as seen in rats (compare Fig. 3). Very low values were found in the outer third of the inner medulla $\left(\mathrm{IMCD}_{1}\right)$. The urea permeability in the inner two thirds of the inner medulla $\left(\mathrm{IMCD}_{2}\right.$ and $\left.\mathrm{IMCD}_{3}\right)$ was 10 -fold higher.

Urea permeability of rabbit papillary surface epithelium. Urea permeability was measured isotopically in five isolated papillary surface epithelial preparations (Table III). The permeability was low $\left(1 \times 10^{-5} \mathrm{~cm} / \mathrm{s}\right)$ both in the absence of and in the presence of arginine vasopressin $\left(10^{-8} \mathrm{M}\right)$.

The tissue that was mounted in the chamber consisted of 
Table I. Descriptive Summary of Urea Permeability Experiments in Rat Inner Medullary Collecting Ducts and Cortical Collecting Ducts*

\begin{tabular}{|c|c|c|c|c|c|c|c|c|c|}
\hline \multirow[b]{2}{*}{ Segment $(n)$} & \multirow[b]{2}{*}{ Length } & \multirow[b]{2}{*}{$\begin{array}{l}\text { Outer } \\
\text { diameter }\end{array}$} & \multirow[b]{2}{*}{$\begin{array}{l}\text { Inner } \\
\text { diameter }\end{array}$} & \multirow[b]{2}{*}{$\begin{array}{l}\text { Collection } \\
\text { rate }\end{array}$} & \multirow[b]{2}{*}{$\begin{array}{l}\text { Potential } \\
\text { difference }\end{array}$} & \multicolumn{3}{|c|}{ Urea concentration } & \multirow[b]{2}{*}{$\begin{array}{l}\text { Urea } \\
\text { permeability }\end{array}$} \\
\hline & & & & & & Perfused & Bath & Collected & \\
\hline & $\mathrm{mm}$ & $\mu m$ & $\mu m$ & $n l / \min$ & $m V$ & $m M$ & $m M$ & $m M$ & $\mathrm{~cm} / \mathrm{s} \times 10^{-5}$ \\
\hline \multirow{2}{*}{$\begin{array}{l}\mathrm{IMCD}_{1} \\
\text { (5) }\end{array}$} & 0.82 & 29 & 21 & 8.6 & 0.1 & 0.0 & 5.1 & 0.41 & 1.7 \\
\hline & \pm 0.14 & \pm 1 & \pm 1 & \pm 4.9 & \pm 0.7 & \pm 0.0 & \pm 0.0 & \pm 0.29 & \pm 0.9 \\
\hline \multirow{2}{*}{$\begin{array}{l}\mathrm{IMCD}_{2} \\
\text { (3) }\end{array}$} & 0.43 & 37 & 24 & 26.4 & 0.0 & 0.0 & 5.1 & 1.53 & 46.6 \\
\hline & \pm 0.02 & \pm 7 & \pm 5 & \pm 9.3 & $\pm 0,0$ & \pm 0.0 & \pm 0.0 & \pm 0.14 & \pm 18.2 \\
\hline \multirow{2}{*}{$\begin{array}{l}\mathrm{IMCD}_{3} \\
\text { (3) }\end{array}$} & 0.41 & 40 & 29 & 35.1 & -0.1 & 0.0 & 5.0 & 1.16 & 39.1 \\
\hline & \pm 0.16 & \pm 7 & \pm 7 & \pm 18.2 & \pm 0.2 & \pm 0.0 & \pm 0.0 & \pm 0.36 & \pm 6.3 \\
\hline $\mathrm{CCD}$ & 0.42 & 38 & 24 & 5.0 & 0.8 & 0.0 & 5.0 & 0.16 & 0.9 \\
\hline (3) & \pm 0.11 & \pm 4 & \pm 5 & \pm 1.5 & \pm 0.8 & \pm 0.0 & \pm 0.0 & \pm 0.05 & \pm 0.4 \\
\hline
\end{tabular}

* Values are given as mean $\pm \mathrm{SD}$. Permeability in $\mathrm{IMCD}_{1}$ was significantly lower than in $\mathrm{IMCD}_{2}$ or $\mathrm{IMCD}_{3}$ by analysis of variance $(P<0.01)$ using Bonferroni's method for contrasts (see text).

the papillary surface epithelium and a thin layer of underlying material made up of interstitial matrix, renal tubules, and blood vessels (See Methods). To determine whether the underlying substratum was rate limiting for urea permeation across the tissue, the epithelium was removed by gently abrading it with a piece of fine nylon mesh (leaving intact the substratum) and the permeability of the substratum was measured with $\left[{ }^{14} \mathrm{C}\right]$ urea. The tissue was observed using high resolution Nomarski phasecontrast microscopy (31) to assure that this procedure effectively removes the papillary surface epithelium while the underlying substratum remains. In four such preparations, the urea permeability of the substratum was $11.7 \pm 1.6 \times 10^{-5} \mathrm{~cm} / \mathrm{s}(P$ $<0.001$ vs. intact epithelium in absence of vasopressin). Because the urea permeability of the substratum was 12-fold greater than the intact tissue, we conclude that the papillary surface epithelium is the rate limiting barrier to urea permeation.

Surface areas of inner medullary collecting ducts and papillary surface epithelium in rat. The surface areas of collecting ducts and papillary surface epithelium were determined by morphometric analysis of histological sections from rat renal papillas. Fig. 5 plots the surface areas per unit papillary length against the distance from the papillary tip. We obtained complete cross sections of the papilla for a length of $\sim 2.25 \mathrm{~mm}$ (Fig. 5) or $45 \%$ of the total inner medullary length. As Fig. 5 shows, the total epithelial surface area of inner medullary collecting ducts in the papilla $\left(86 \mathrm{~mm}^{2}\right)$ was 10 -fold greater than the total area of the papillary surface epithelium $\left(8.7 \mathrm{~mm}^{2}\right)$. The ratio of the two surface areas (collecting ducts to papillary surface epithelium) increases with distance from the papillary tip.

Surface areas of inner medullary collecting ducts and papillary surface epithelium in rabbit. The surface areas of collecting ducts and papillary surface epithelium were also determined in rabbit renal papillas. Fig. 6 plots the surface areas per unit papillary length against the distance from the papillary tip. We could obtain complete papillary cross sections for a distance of $\sim 1.25$ $\mathrm{mm}$ from the papillary tip (Fig. 6 ) or $\sim 20 \%$ of the total inner

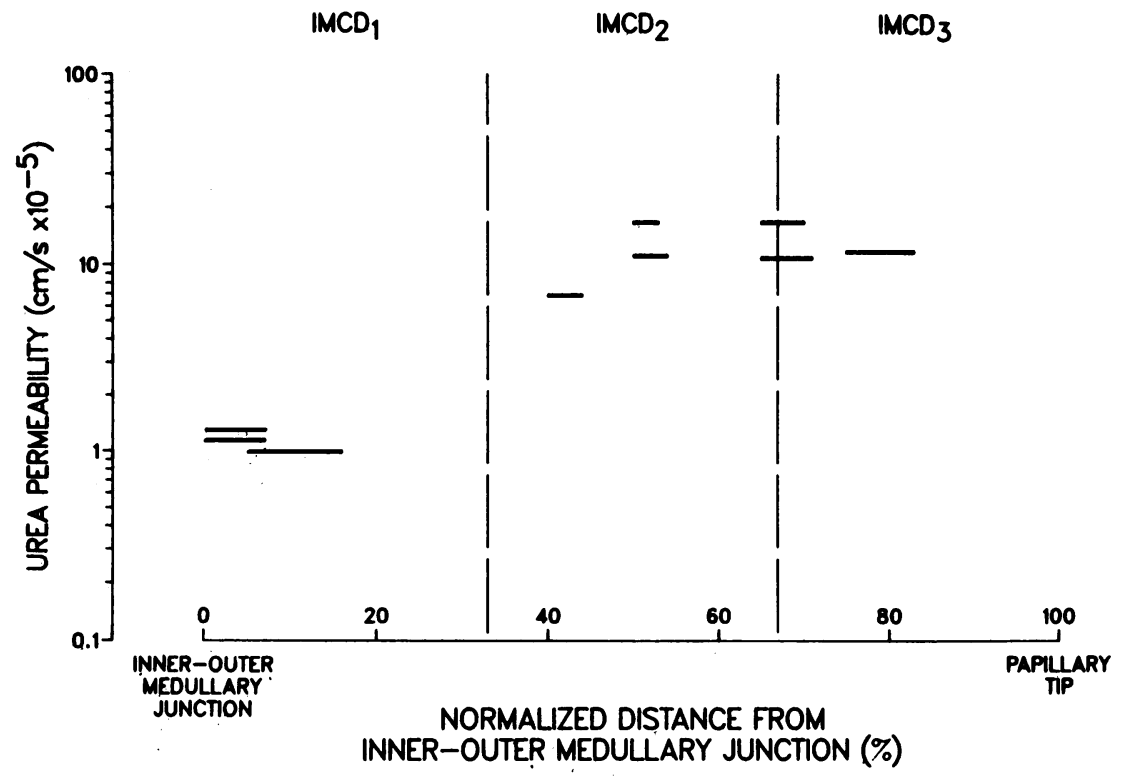

Figure 4. Rabbit inner medullary collecting ducts. Urea permeabilities in rabbit inner medullary collecting ducts as a function of distance along inner medullary axis. The mean permeability for each tubule is represented by a horizontal line indicating the original position of the tubule in the inner medulla. Solid lines represent permeabilities measured with a 5-mM bath-to-lumen urea gradient. To allow statistical comparisons, tubules were arbitrarily divided into three groups based on the origin of the tubules in the medulla: $\mathrm{IMCD}_{1}, \mathrm{IMCD}_{2}$, and $I M C D_{3}$. Permeability in $I M C D_{1}$ was significantly lower than in $\mathrm{IMCD}_{2}$ or $\mathrm{IMCD}_{3}$ by analysis of variance $(P<0.01$ using Bonferroni's method for contrasts [41]. See Table II for full descriptive summary of experiments. 
Table II. Descriptive Summary of Urea Permeability Experiments in Rabbit Inner Medullary Collecting Ducts*

\begin{tabular}{|c|c|c|c|c|c|c|c|c|c|}
\hline \multirow[b]{2}{*}{ Segment $(n)$} & \multirow[b]{2}{*}{ Length } & \multirow[b]{2}{*}{$\begin{array}{l}\text { Outer } \\
\text { diameter }\end{array}$} & \multirow[b]{2}{*}{$\begin{array}{l}\text { Inner } \\
\text { diameter }\end{array}$} & \multirow[b]{2}{*}{$\begin{array}{l}\text { Collection } \\
\text { rate }\end{array}$} & \multirow[b]{2}{*}{$\begin{array}{l}\text { Potential } \\
\text { difference }\end{array}$} & \multicolumn{3}{|c|}{ Urea concentration } & \multirow[b]{2}{*}{$\begin{array}{l}\text { Urea } \\
\text { permeability }\end{array}$} \\
\hline & & & & & & Perfused & Bath & Collected & \\
\hline & $\mathrm{mm}$ & $\mu m$ & $\mu m$ & $n l / m i n$ & $m V$ & $m M$ & $m M$ & $m M$ & $\mathrm{~cm} / \mathrm{s} \times 1 \sigma^{-s}$ \\
\hline $\mathrm{IMCD}_{1}$ & 0.93 & 38 & 21 & 4.1 & 1.1 & 0.0 & 5.3 & 0.55 & 1.2 \\
\hline (3) & \pm 0.22 & \pm 3 & \pm 3 & \pm 0.5 & \pm 0.3 & \pm 0.0 & \pm 0.0 & \pm 0.15 & \pm 0.1 \\
\hline $\mathrm{IMCD}_{2}$ & 0.37 & 73 & 49 & 18.8 & 0.3 & 0.0 & 5.0 & 1.03 & 11.6 \\
\hline (3) & \pm 0.07 & \pm 20 & \pm 10 & \pm 12.0 & \pm 0.4 & \pm 0.0 & \pm 0.0 & \pm 0.42 & \pm 4.9 \\
\hline $\mathrm{IMCD}_{3}$ & 0.64 & 81 & 41 & 37.6 & 1.0 & 0.0 & 5.1 & 0.86 & 13.1 \\
\hline (3) & \pm 0.14 & \pm 17 & \pm 15 & \pm 19.1 & \pm 0.9 & \pm 0.0 & \pm 0.0 & \pm 0.32 & \pm 3.2 \\
\hline
\end{tabular}

* Values are given as mean $\pm \mathrm{SD}$. Permeability in $\mathrm{IMCD}_{1}$ was significantly lower than in $\mathrm{IMCD}_{2}$ or $\mathrm{IMCD}_{3}$ by analysis of variance $(P<0.01)$ using Bonferroni's method for contrasts (see text).

medullary length. Fig. 6 shows that the total epithelial surface area of inner medullary collecting ducts in this region was approximately threefold greater than the area of the papillary surface epithelium. As in the rat, the ratio of the two surface areas (collecting ducts to papillary surface epithelium) increases with distance from the papillary tip.

\section{Discussion}

Maintenance of a high concentration of urea in the inner medullary interstitium depends on sustained entry of urea into the interstitium to replace the urea lost via the vasa recta. Urea has been proposed to enter the inner medullary interstitium via two pathways: $(a)$ passive reabsorption from the inner medullary collecting ducts, and $(b)$ passive transport from the renal pelvic space across the papillary surface epithelium. In this study, we have measured two determinants of passive transport across these epithelia: urea permeability coefficient and epithelial surface area. This information is required for a quantitative comparison of urea transport across the two pathways. Several new findings have emerged. First, the inner medullary collecting duct of both rat and rabbit is nonuniform along its length with regard to its permeability to urea. The urea permeability is relatively low in the outer third of the inner medulla, but rises to extremely high values in the ininer two-thirds of the inner medulla. Second, the urea permeability of the papillary surface epithelium is very low relative to that of the inner medullary collecting ducts at the

Table III. Urea Permeability of Rabbit Papillary Surface Epithelium*

\begin{tabular}{llcl}
\hline & Potential difference & Resistance & Urea permeability \\
\hline & $\mathrm{mV}$ & $\mathrm{ohm} \cdot \mathrm{cm}^{2}$ & $\mathrm{~cm} / \mathrm{s} \times 10^{-\mathrm{s}}$ \\
$\begin{array}{l}\text { Control } \\
\text { Vasopressin }\end{array}$ & $0.0 \pm 0.0$ & $95.8 \pm 6.7$ & $1.09 \pm 0.15$ \\
$(10 \mathrm{nM})$ & $0.0 \pm 0.0$ & $102.4 \pm 7.2$ & $1.03 \pm 0.19$ \\
\hline
\end{tabular}

* Values are mean $\pm \mathrm{SE}$ for five experiments. Values with vasopressin in basolateral perfusate were not significantly different from control values. Urea permeability of underlying tissue devoid of papillary surface epithelium was $11.7 \pm 1.6 \times 10^{-5} \mathrm{~cm} / \mathrm{s}(P<0.001 \mathrm{vs}$. intact epithelium; see text). same level of the inner medulla. Third; the epithelial surface area of the papillary surface epithelium is small relative to that of the inner medullary collecting ducts in the papillas of both species. The physiological significance of these findings will be discussed in the following.

Axial heterogeneity along the inner medullary collecting duct. Morphological observations provided the first clues that the inner medullary collecting ducts are not uniform throughout the inner medulla. There is a progressive increase in tubule diameter and mean cell height from the inner-outer medullary junction to the papillary tip (18). This is associated with clearcut changes in principal cell morphology $(19,20)$. In addition, inner medullary collecting ducts from the outer third of the inner medulla contain both intercalated and principal cells $(20)$. This contrasts with the remainder of the inner medullary collecting duct, which contains only principal cells.

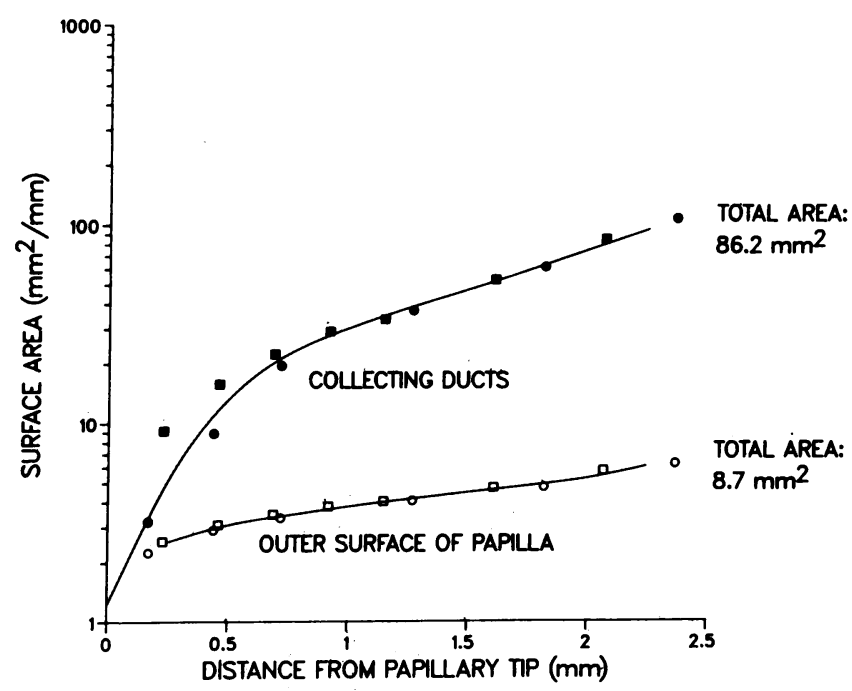

Figure 5. Rat renal papilla. Surface areas of inner medullary collecting ducts and papillary surface epithelium in rat renal papilla. Vertical axis is surface area per unit distance along long axis of papilla, i.e., total circumference of collecting ducts and surface epithelium. Total areas (shown at right) were obtained by integrating over entire length of papilla. Data from two rats are shown as indicated by squares and circles. 


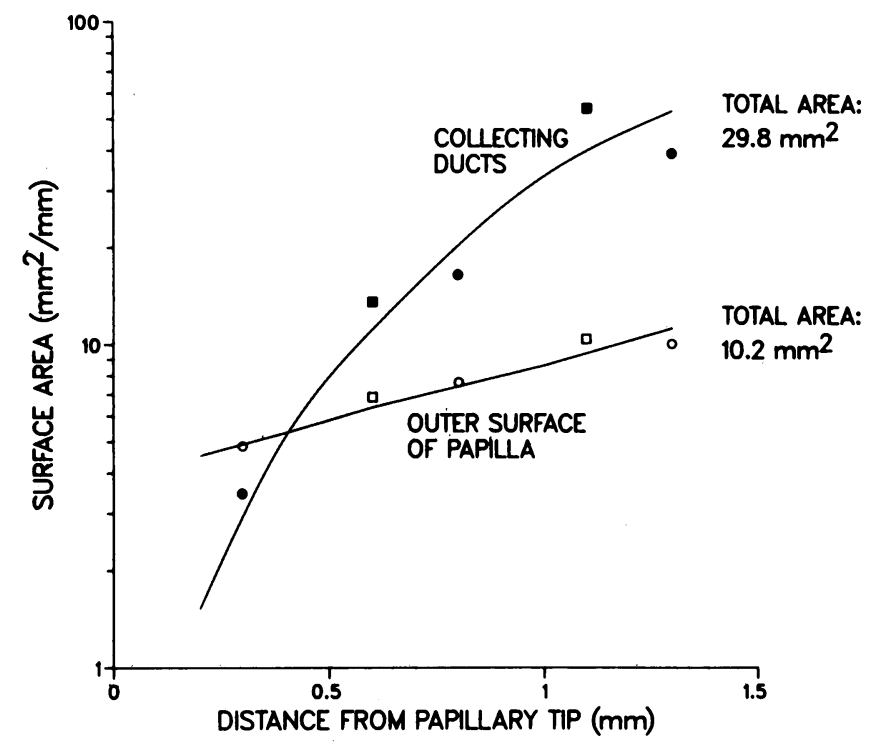

Figure 6. Rabbit renal papilla. Surface areas of inner medullary collecting ducts and papillary surface epithelium in rabbit renal papilla. Vertical axis is surface area per unit distance along long axis of papilla, i.e., total circumference of collecting ducts and surface epithelium. Total areas (shown at right) were obtained by integrating over entire length of papilla. Data from two rabbits are shown as indicated by squares and circles.

The hypothesis that the inner medullary collecting duct may be nonuniform with respect to urea permeability arose from mathematical modelling studies $(34,35)$. These studies, using a detailed systems model of the concentrating mechanism, showed that when a uniformly high urea permeability was assumed along the inner medullary collecting duct (with a step transition from a low to a high value at the inner-outer medullary junction), rapid absorption of urea from the early inner medullary collecting duct was predicted, leaving little urea in the final urine and allowing very little urea accumulation in the inner medulla. In contrast, when a gradual rise in urea permeability to high values in the inner half of the inner medulla was assumed, high concentrations of urea in the final urine and inner medullary interstitium were predicted by the model. That is, urea absorption from the inner medullary collecting ducts must be delayed to the deepest part of the inner medulla (where the effective blood flow is lowest) to achieve maximal urea concentrations in the inner medullary interstitium.

The measurements of urea permeability accomplished in the present studies confirmed the predictions of the prior mathematical modelling studies. In both rat (Fig. 3) and rabbit (Fig. 4), the urea permeability was found to be relatively low in collecting ducts from the outer third of the inner medulla, but increased to very high values in collecting ducts from the inner two-thirds of the inner medulla. The permeability in the inner two-thirds is highest among all renal tubule segments. Accurate measurement of the permeability required use of very rapid perfusion rates to avoid complete equilibration between the bath and luminal fluid (Tables I and II). Although not tested in this study, previous studies indicate that the urea permeability can increase to an even higher value in response to vasopressin (14, 15). Given the magnitude of the permeability, it is likely that urea fluxes from lumen to interstitium are very rapid in vivo.

A previous study by Rocha and Kokko (16) reported a mean urea permeability coefficient of $2.3 \times 10^{-5} \mathrm{~cm} / \mathrm{s}$ in the rabbit inner medullary collecting duct. This value was approximately an order of magnitude lower than values reported for the rat inner medullary collecting duct $(14,15)$, which raised the possibility that there is an important difference between rabbit and rat with regard to urea permeability of the inner medullary collecting duct (17). The data from the present study indicate that the lower value in the rabbit was due to a relatively more proximal origin of the dissected segments rather than to any striking difference in the two species. The median diameter of the inner medullary collecting duct segments studied by Rocha and Kokko was $33.5 \mu \mathrm{m}$, which is consistent with the diameter of the IMCD segments in the present study, but is much smaller than segments from the inner two-thirds of the inner medulla (Table II). Furthermore, the mean urea permeability coefficient reported by Rocha and Kokko is in close accord with the mean value found in IMCD $_{1}$ segment in the present study.

Although the general profile of urea permeability along the inner medullary duct of rat and rabbit is similar, a quantitative comparison reveals that the urea permeability coefficients tend to be moderately lower in the rabbit tubules. It should be noted however that the diameter of the rabbit inner medullary collecting duct is substantially greater than that of the rat in the inner two-thirds of the inner medulla (reference 18 and Tables I and II). Consequently, the urea permeability-surface area product is quite similar in inner medullary collecting ducts of rats and rabbits.

This is the first study in which inner medullary collecting ducts from defined locations along the entire length of the inner medulla of both rat and rabbit have been perfused in vitro. Accordingly, it seems appropriate to comment on the technical aspects of the experiments. We can draw three important conclusions regarding the technique: (a) Inner medullary collecting ducts from rats were much easier to dissect and perfuse than inner medullary collecting ducts from rabbits, particularly in the inner two-thirds of the inner medulla. Because of their very large diameters, the ducts from rabbits tended to split down the middle rather than to come out intact. It frequently required a very long period of dissection to obtain suitable tubules from rabbits. In contrast, rat inner medullary collecting ducts could almost always be obtained rapidly. (b) Treatment of the animals with furosemide greatly improved the quality of the dissected tubules. We propose that, by washing out the corticomedullary osmolality gradient in vivo, furosemide diuresis prevents the osmotic shock that might otherwise occur when the tissue is placed in the dissection medium. In addition, the tubules are more readily visible in the inner medulla after furosemide diuresis because the tubule lumens remain open. (c) The length of the perfused segments is limited by the frequency of junctions between collecting ducts as the collecting duct system converges in the inner medulla of the rat. There are, however, unbranched segments of at least $0.5-1.0 \mathrm{~mm}$ in all three regions of the inner medulla. This range is similar to the lengths of rat cortical collecting ducts found practical for in vitro perfusion in previous studies.

Papillary surface epithelium. The apparent morphological similarity between the papillary surface epithelium and the inner medullary collecting duct $(36,37)$ has led to the widespread assumption that the two epithelia may have similar functional properties. Consequently, it has been widely assumed that the urea permeability of the papillary surface epithelium is high and would allow a rapid flux of urea from the pelvic space to the 
papillary interstitium. However, our direct measurements in the papillary surface epithelia isolated from the renal medulla and mounted in a perfusion chamber revealed that the urea permeability is at least 10 -fold lower than that of the papillary part of the inner medullary collecting duct (Table III). Thus, we conclude that the papillary surface epithelium is not simply an extension of the inner medullary collecting duct, but is functionally distinct.

A major objective of this study was to obtain permeability and surface area data necessary to test the hypothesis that passive urea transport across the papillary surface epithelium can be an important source of urea for accumulation in the inner medullary interstitium. For passive urea transport across the papillary surface epithelium to contribute significantly to urea addition to the inner medullary interstitium, the rate of transport across this epithelium must be at least a substantial fraction of the rate of passive urea absorption from the inner medullary collecting ducts, which are already known to absorb urea at a considerable rate $(15-30 \%$ of the filtered load $[12,13])$. In the following, we use the measurements made in this study to calculate the probable rate of urea transport across the papillary surface epithelium relative to the rate of urea absorption from the inner medullary collecting ducts.

The rate of passive transport of a nonelectrolyte across an epithelium is equal to the product of three values: the permeability, the epithelial surface area, and the concentration difference across the epithelium. In this study, we have measured two of these factors in both papillary surface epithelium and in inner medullary collecting ducts. The values obtained are compared in Table IV. We found that the urea permeability of the rabbit papillary surface epithelium is approximately one-twelfth that of the inner medullary collecting ducts in the inner two-thirds of the inner medulla $\left(\mathrm{IMCD}_{2}\right.$ and $\left.\mathrm{IMCD}_{3}\right)$. Furthermore, the epithelial surface area of the rabbit papillary surface epithelium was approximately one-third that of the inner medullary collecting ducts. Therefore, in the rabbit the product of the urea permeability and surface area of the papillary surface epithelium is no greater than $1 / 36$ th that of the inner medullary collecting

Table IV. Comparison of Urea Permeability-Surface Area Products in Collecting Ducts and Papillary Surface Epithelium from Papillary Portion of the Inner Medulla*

\begin{tabular}{|c|c|c|c|c|c|c|}
\hline & \multicolumn{2}{|c|}{$\begin{array}{l}\text { Urea } \\
\text { permeability } \\
\left(P_{u}\right)\end{array}$} & \multicolumn{2}{|c|}{$\begin{array}{l}\text { Surface area } \\
\left(S_{a}\right)^{*}\end{array}$} & \multicolumn{2}{|l|}{$P_{u} \times S_{\mathrm{a}}$} \\
\hline & Rabbit & Rat & Rabbit & Rat & Rabbit & Rat \\
\hline & \multicolumn{2}{|c|}{$\times 10^{-5} \mathrm{~cm} / \mathrm{s}$} & $m m^{2}$ & $m m^{2}$ & $\mathrm{~mm}^{3} / \mathrm{min}$ & $\mathrm{mm}^{3} / \min$ \\
\hline \multicolumn{7}{|l|}{ Collecting ducts } \\
\hline$\left(\mathrm{IMCD}_{2}+\mathrm{IMCD}_{3}\right)$ & 12 & 43 & 30 & 86 & 2.16 & 22.3 \\
\hline \multicolumn{7}{|l|}{ Papillary surface } \\
\hline epithelium (PSE) & 1 & - & 10 & 9 & 0.06 & - \\
\hline IMCD/PSE ratio & 12 & - & 3 & 10 & 36 & 一 \\
\hline
\end{tabular}

Urea permeability values are means from Tables I-III.

Surface area values are from Figs. 5 and 6.

* For the purposes of this paper, the papillary portion is assumed to include the inner two-thirds of the inner medulla.

₹ The surface areas could not be determined for the entire papilla (see Figs. 5 and 6). Because the IMCD/PSE surface area ratios increase toward the outer medulla, the values given here presumably underestimate the ratios for the entire papilla. ducts. This means that, in the rabbit, the transepithelial concentration gradient across the papillary surface epithelium would have to be at least 36-fold larger than that across the inner medullary collecting ducts to have the same overall rate of urea transport. In the rat, the inner medullary collecting duct urea permeability is even larger than in the rabbit and the papillary surface epithelium:collecting duct surface area ratio is smaller than in the rabbit (Table IV), which suggests that the urea permeability-surface area product of the papillary surface epithelium may be low relative to that of the inner medullary collecting duct in the rat as in the rabbit. However, we were unable to obtain large enough sheets of papillary surface epithelium from the rat to measure its urea permeability and thus we cannot make a complete comparison of papillary surface epithelium and inner medullary collecting duct in this species.

Direct measurements of urea concentration gradients across the papillary surface epithelium are not available. However, urea concentration gradients across the inner medullary collecting duct epithelium have been measured. Micropuncture measurements in nondiuretic rats $(5)$ revealed that the ratio of urea concentrations in inner medullary collecting ducts to vasa recta plasma water was 1.24 when the mean collecting duct urea concentration was $345 \mathrm{mM}$. From this, we calculate that the mean urea concentration difference across the inner medullary collecting duct was $\sim 70 \mathrm{mM} .^{2}$ It is unlikely that the urea concentration difference across the papillary surface epithelium is substantially greater, particularly in view of the fact that the urea concentration in the pelvic urine of nondirectic rats was observed to be $\sim 40 \%$ lower adjacent to the papillary base than near the papillary tip (25). Thus, the actual transepithelial urea concentration gradient is likely to be far short of that required for a physiologically significant flux across the papillary surface epithelium. If we make the generous estimate that the concentration gradient across the papillary surface epithelium is on an average 1.5-3 times that across the inner medullary collecting ducts and assume that the urea permeability-surface area product of the inner medullary collecting ducts is 36 -fold greater than that of the papillary surface epithelium (Table IV), we estimate that the total rate of passive urea transport across the papillary surface epithelium can be no greater than $4-8 \%$ of that across the inner medullary collecting ducts.

The possibility of physiologically significant urea transport from the renal pelvis has also been investigated in two studies using micropuncture techniques $(13,25)$. Marsh and Martin (13) measured urea exit from the pelvic space indirectly by comparing the mass flow rate of urea reaching the late portion of the ureter with that in the inner medullary collecting ducts at the papillary tip and concluded that there was no significant flux

2. To test whether a 70-mM urea concentration gradient across the inner medullary collecting ducts is realistic, we have calculated the expected rate of urea absorption from this gradient $(\delta C)$, the urea permeability $\left(P_{\mathrm{u}}\right)$ and the total surface area of collecting ducts $\left(A_{\mathrm{s}}\right)$ using the equation $J_{\mathrm{u}}=A_{\mathrm{s}} \cdot P_{\mathrm{u}} \cdot \delta \mathrm{C}$. Using values for the rat, $A_{\mathrm{s}}=86.2 \mathrm{~mm}^{2}$ (Fig. 5) and $P_{\mathrm{u}}$ $=39 \times 10^{-5} \mathrm{~cm} / \mathrm{s}\left(\right.$ Table I, $\left.\mathrm{IMCD}_{3}\right)$, and $\delta \mathrm{C}=70 \mathrm{mM}$, the calculated rate of urea absorption from the inner medullary collecting ducts is 1.4 $\times 10^{-3} \mathrm{mmol} / \mathrm{min}$. If this value is compared with the filtered load of urea in normal rats $\left(6 \times 10^{-3} \mathrm{mmol} / \mathrm{min}\right.$ [38]), the predicted urea absorption from the inner medullary collecting ducts will be $1.4 / 6 \times 100 \%$ $=23 \%$ of the filtered load. This value is consistent with the range observed $(15-30 \%)$ in micropuncture and microcatherization studies $(12,13)$, indicating that the assumed $70 \mathrm{mM}$ concentration gradient is realistic. 
of urea out of the pelvic space. In more recent studies by Bargman et al. (25), the fraction of filtered urea reaching the urinary bladder $(28 \%)$ was found to be significantly lower than the fraction of filtered urea in the pelvic urine near the papillary tip (34\%) collected by micropuncture through an intact pelvic wall. Mathematical analysis of data obtained from direct micropuncture of the renal pelvic space and microcatherization of the pelvic fornices (25) led to the conclusion that some efflux of urea into the medullary interstitium must have occurred. It is probable that most of the urea efflux was into the outer medulla rather than into the inner medulla, considering the large surface area of the outer medulla that is exposed to pelvic urine relative to that of the inner medulla $(39,40)$. Therefore, the results of Bargman et al. (25) are not in direct conflict with the conclusion from the present study that urea transport from the pelvic space across the papillary surface epithelium is unlikely to be a major source of urea for accumulation in the inner medullary interstitium.

Evidence usually cited as favoring the hypothesis that the renal pelvis may be an important source of urea supply to the inner medullary interstitium comes from studies utilizing superperfusion of the pelvis with urea-containing solutions (2123). The results of these pelvic superfusion studies are consistent with the view that urea can penetrate the papillary surface epithelium given sufficient contact time and a sufficient concentration gradient. The urea permeability of the papillary surface epithelium measured in the present study $\left(1.2 \times 10^{-5} \mathrm{~cm} / \mathrm{s}\right)$ is large enough to account for substantial urea transfer when a large urea concentration gradient is maintained over a relatively long period of time as in the pelvic superfusion studies. That is, although the papillary surface epithelium has a urea permeability that is low relative to the inner medullary collecting ducts, the epithelium is certainly not entirely impermeable to urea. Thus, the permeability value measured in the present study is compatible with the findings of the superfusion studies, and yet it is too low to account for a physiologically important urea flux across the papillary surface under normal steady-state conditions.

Summary. The findings of the present study do not support the proposal that passive urea transport across the papillary surface epithelium of the mammalian kidney is an important source of urea to the inner medullary interstitium. Both the urea permeability and the epithelial surface area of the papillary surface epithelium are small compared to corresponding values for the collecting ducts in the renal papilla. An additional important finding of the present study is that the inner medullary collecting duct is functionally heterogeneous. The outer third of the inner medullary collecting duct $\left(\mathrm{IMCD}_{1}\right)$ has a low permeability to urea. In contrast, the urea permeability of the inner two thirds of the inner medullary collecting duct $\left(\mathrm{IMCD}_{2}\right.$ and $\left.\mathrm{IMCD}_{3}\right)$ is remarkably high, which suggests the presence of a facilitated urea transport pathway.

\section{Acknowledgments}

We thank Drs. Maurice Burg and Michael Horster for critically reading the manuscript.

\section{References}

1. Murdaugh, H. V., Jr., B. Schmidt-Nielsen, E. M. Doyle, and R. O'Dell. 1958. Renal tubular regulation of urea excretion in man. J. Appl. Physiol. 13:263-268.
2. Ullrich, K. J., and K. H. Jarausch. 1956. Untersuchungen zum Problem der Harnkonzentrierung und Harnverdunnung. Pfluegers Arch. Eur. J. Physiol. 262:537-550.

3. Truniger, B., and B. Schmidt-Nielsen. 1964. Intrarenal distribution of urea and related compounds: effects of nitrogen intake. Am. J. Physiol. 207:971-978.

4. Gunther, R. A., and L. Rabinowitz. 1980. Urea and renal concentrating ability in the rabbit. Kidney Int. 17:205-222.

5. Ullrich, K. J., G. Rumrich, and B. Schmidt-Nielsen. 1967. Urea transport in the collecting duct of rats on normal and low protein diet. Pfluegers Arch. Eur. J. Physiol. 295:147-156.

6. Marsh, D. J. 1970. Solute and water flows in thin limbs of Henle's loop in the hamster kidney. Am. J. Physiol. 218:824-831.

7. Pennell, J. P., V. Sanjana, N. R. Frey, and R. L. Jamison. 1975. The effect of urea infusion on the urinary concentrating mechanism in protein-depleted rats. J. Clin. Invest. 55:399-409.

8. Imbert, M., and C. de Rouffignac. 1976. Role of sodium and urea in the renal concentrating mechanism in Psammomys obesus. Pfluegers Arch. Eur. J. Physiol. 361:107-114.

9. Berliner, R. W., N. G. Levinsky, D. G. Davidson, and M. Eden. 1958. Dilution and concentration of the urine and the action of antidiuretic hormone. Am. J. Med. 27:730-744.

10. Stephenson, J. L. 1972. Concentration of urine in a central core model of the renal counterflow system. Kidney Int. 2:85-94.

11. Kokko, J. P., and F. C. Rector. 1972. Countercurrent multiplication system without active transport in inner medulla. Kidney Int. 2: 214-223.

12. Kluemper, J. D., K. J. Ullrich, and H. H. Hilger. 1958. Das Verhalten des Harnstoff in den Sammelrohren der Säugetierniere. Pfluegers Arch. Eur. J. Physiol. 267:238-243.

13. Marsh, D. J., and C. M. Martin. 1980. Lack of water or urea movement from pelvic urine to papilla in hydropenic hamsters. Min. Electrolyte Metab. 3:81-86.

14. Morgan, T., and R. W. Berliner. 1968. Permeability of the loop of Henle, vasa recta, and collecting duct to water, urea, and sodium. Am. J. Physiol. 215:108-115.

15. Rocha, A. S., and L. H. Kudo. 1982. Water, urea, sodium, chloride, and potassium transport in the in vitro isolated perfused papillary collecting duct. Kidney Int. 22:485-491.

16. Rocha, A. S., and J. P. Kokko. 1974. Permeability of medullary nephron segments to urea and water: effect of vasopressin. Kidney Int. 6:379-387.

17. Rector, F. C., Jr. 1977. Renal concentrating mechanisms. In Disturbances in Body Fluid Osmolality. T. E. Andreoli, J. J. Grantham, and F. C. Rector, Jr., editors. American Physiological Society, Bethesda, MD 179-196.

18. Knepper, M. A., R. A. Danielson, G. M. Saidel, and R. S. Post. 1977. Quantitative analysis of renal medullary anatomy in rats and rabbits. Kidney Int. 12:313-323.

19. Jamison, R. L., and W. Kriz. 1982. Urinary Concentrating Mechanism: Structure and Function. Oxford University Press, Oxford 189-205.

20. Madsen, K. M., and C. C. Tisher. 1986. Structural-functional relationship along the distal nephron. Am. J. Physiol. 250:F1-F15.

21. Schmidt-Nielsen, B., and H. D. Pagel. 1968. Mechanism of urea retention in the renal medulla. In Urea and the Kidney. B. SchmidtNielsen and D. N. S. Kerr, editors. Excerpta Medica, Amsterdam. 393400.

22. Schuetz, W., and J. Schnermann. 1972. Pelvic urine composition as a determinant of inner medullary solute concentration and urine osmolality. Pfluegers Arch. Eur. J. Physiol. 334:154-166.

23. Bonventre, J. V., R. J. Roman, and C. Lechene. 1980. Effect of urea concentration of pelvic fluid on renal concentrating ability. Am. J. Physiol. 239:F609-F610.

24. Lory, P., A. Gilg, and M. Horster. 1983. Renal countercurrent system: role of collecting duct convergence and pelvic urea predicted from a mathematical model. J. Math. Biol. 16:281-304. 
25. Bargman, J., S. L. Leonard, E. McNeely, C. Robertson, and R. L. Jamison. 1984. Examination of transepithelial exchange of water and solute in the rat renal pelvis. J. Clin. Invest. 74:1860-1870.

26. Burg, M. B. 1972. Perfusion of isolated renal tubules. Yale $J$. Biol. Med. 45:321-326.

27. Good, D. W., and G. G. Vurek. 1983. Picomole quantitation of ammonia by flow-through fluorometry. Anal. Biochem. 130:199-202.

28. Knepper, M. A., D. W. Good, and M. B. Burg. 1985. Ammonia and bicarbonate transport by rat cortical collecting ducts perfused in vitro. Am. J. Physiol. 249:F870-F877.

29. Burg, M. B., and N. Green. 1973. Function of the thick ascending limb of Henle's loop. Am. J. Physiol. 224:659-668.

30. Helman, S. I., and D. A. Miller. 1971. In vitro techniques for avoiding edge damage in studies of frog skin. Science (Wash. DC). 173: 146-148.

31. Sands, J. M., M. A. Knepper, and K. R. Spring. 1986. Na-K-Cl cotransport in apical membrane of rabbit renal papillary surface epithelium. Am. J. Physiol. 251:F475-F484.

32. Kreyszig, E. 1967. Advanced Engineering Mathematics. 2nd ed. J. P. Wiley \& Sons, New York. 31-38.
33. Knepper, M. A. 1983. Urea transport in isolated thick ascending limbs and collecting ducts from rats. Am. J. Physiol. 245:F634-F639.

34. Chandhoke, P. S., and G. M. Saidel. 1981. Mathematical model of mass transport throughout the kidney: effects of nephron heterogeneity and tubular-vascular organization. Ann. Biomed. Eng. 9:263-301.

35. Chandhoke, P. S., G. M. Saidel, and M. A. Knepper. 1985. Role of inner medullary collecting duct $\mathrm{NaCl}$ transport in urinary concentration. Am. J. Physiol. 249:F688-F697.

36. Pfeiffer, E. W. 1968. Comparative anatomical observations of the mammalian renal pelvis and medulla. J. Anat. 102:321-331.

37. Bonventre, J. V., M. J. Karnovsky, and C. P. Lechene. 1978. Renal papillary epithelial morphology in antidiuresis and water diuresis. Am. J. Physiol. 235:F69-F76.

38. Roch-Ramel, F., F. Chomety, and G. Peters. 1968. Urea concentrations in tubular fluid and in renal tissue of nondiuretic rats. $\mathbf{A m}$. J. Physiol. 215:429-438.

39. Lacy, E. R., and B. Schmidt-Nielsen. 1979. Anatomy of the renal pelvis of the hamster. Am. J. Anat. 154:291-320.

40. Lacy, E. R. 1980. The mammalian renal pelvis: physiological implications from morphometric analysis. Anat. Embryol. 160:131-144. 